\title{
On ISS and iISS properties of homogeneous systems
}

\author{
Emmanuel Bernuau, Andrey Polyakov, Denis Efimov and Wilfrid Perruquetti
}

\begin{abstract}
Several conditions are proposed to check ISS and iISS properties for generic nonlinear systems applying the weighted homogeneity concept (global or local). The advantages of this result is that, under some mild conditions, the system robustness can be established as a function of the degree of homogeneity.
\end{abstract}

\section{INTRODUCTION}

The problem of robustness and stability analysis with respect to external inputs (like exogenous disturbances or measurement noises) for dynamical systems is in the center of attention of many researches [1], [2], [3], [4], [5], [6]. One of the most popular theories, which can be used for this robustness analysis for nonlinear systems, was originated more than 20 years ago [7] and it is based on the Input-to-State Stability (ISS) property and many related notions (see a recent survey [8]). The advantages of ISS theory include a complete list of necessary and sufficient conditions, existence of the Lyapunov method extension, a rich variety of stability concepts adopted for different control and estimation problems.

The main tool to check the ISS property for a nonlinear system consists in a Lyapunov function design, which satisfies some sufficient conditions. As usual, there is no generic approach to select a Lyapunov function for nonlinear systems. Therefore, computationally tractable approaches for ISS verification for particular classes of nonlinear systems are of great practical importance. In this work we are going to propose such a technique for checking ISS and iISS properties for a class of homogeneous and locally homogeneous systems.

Homogeneity is an intrinsic property of an object, which remains consistent with respect to some scaling, e.g. level sets (resp. solutions) are preserved for homogeneous functions (resp. vector fields). The notion of weighted homogeneity was found useful by many authors [9], [10], [11], [12], [13], [14]. The main feature of this property is that it transforms a local (stability) property of the system to the whole state space via a suitably defined scaling. In some cases such a globality of the system behavior becomes ambiguous, that is why the local homogeneity notion has been recently proposed [15], [16]. In this case the property transfer can be carried out on a subspace using different local scales.

The ISS notion of homogeneous systems has been studied in [17], [18], [15]. In this work we are going to generalize

The first and the last two authors are with LAGIS UMR 8219, Universite Lille Nord de France, Ecole Centrale de Lille, Avenue Paul Langevin, BP 48, 59651 Villeneuve d'Ascq, France. The last three authors are with Non-A project at INRIA - LNE, Parc Scientifique de la Haute Borne, 40 avenue Halley, Bât. A Park Plaza, 59650 Villeneuve d'Ascq, France the result of those works and extend it to the integral ISS (iISS) property. The underlying idea of the proposed results is that for a nonlinear system its asymptotic stability with zero disturbance implies a certain robustness (ISS or iISS) under homogeneity conditions. Note that to establish asymptotic stability of a homogeneous system one can use a non-strict Lyapunov function with the Krasovskiy-LaSalle arguments.

The outline of the paper is as follows. Notations used in the paper are given in Section II. The robust stability notions under consideration and homogeneity are introduced in Section III. The ISS and iISS properties of homogeneous systems are studied in Section IV. The same analysis for locally homogeneous systems is done in Section V.

\section{NOTATIONS}

Through the paper the following notations will be used:

- $\mathbb{R}_{+}=\{x \in \mathbb{R}: x \geq 0\}$, where $\mathbb{R}$ is the set of real number.

- $|\cdot|$ denotes the absolute value in $\mathbb{R},\|$.$\| denotes the$ Euclidean norm on $\mathbb{R}^{n}$.

- For a (Lebesgue) measurable function $d: \mathbb{R}_{+} \rightarrow \mathbb{R}^{m}$ define the norm $\|d\|_{\left[t_{0}, t_{1}\right)}=\operatorname{ess} \sup _{t \in\left[t_{0}, t_{1}\right)}\|d(t)\|$, then $\|d\|_{\infty}=\|d\|_{[0,+\infty)}$ and the set of $d(t)$ with the property $\|d\|_{\infty}<+\infty$ we will further denote as $\mathcal{L}_{\infty}$ (the set of essentially bounded measurable functions).

- A continuous function $\alpha: \mathbb{R}_{+} \rightarrow \mathbb{R}_{+}$belongs to the class $\mathcal{K}$ if $\alpha(0)=0$ and the function is strictly increasing. The function $\alpha: \mathbb{R}_{+} \rightarrow \mathbb{R}_{+}$belongs to the class $\mathcal{K}_{\infty}$ if $\alpha \in \mathcal{K}$ and it is increasing to infinity. A continuous function $\beta$ : $\mathbb{R}_{+} \times \mathbb{R}_{+} \rightarrow \mathbb{R}_{+}$belongs to the class $\mathcal{K} \mathcal{L}$ if $\beta(\cdot, t) \in \mathcal{K}_{\infty}$ for each fixed $t \in \mathbb{R}_{+}$and $\lim _{t \rightarrow+\infty} \beta(s, t)=0$ for each fixed $s \in \mathbb{R}_{+}$. For any $\alpha \in \mathcal{K}$ and $s, r \in \mathbb{R}_{+}$, $\alpha(s+r) \leq \alpha(2 s)+\alpha(2 r)$.

- The notation $D V(x) f(x)$ stands for the directional derivative of a continuously differentiable function $V$ with respect to the vector field $f$ evaluated at point $x$.

- Young's inequality: $s v \leq \frac{s^{p}}{p}+\left(1-\frac{1}{p}\right) v^{\frac{p}{p-1}}$ for any $s, v \in$ $\mathbb{R}_{+}$and $p>1$.

- A series of integers $1,2, \ldots, n$ is denoted by $\overline{1, n}$.

\section{PRELIMINARIES}

In this work we will consider the following nonlinear system:

$$
\dot{x}=f(x, d),
$$

where $x \in \mathbb{R}^{n}$ is the state, $d \in \mathbb{R}^{m}$ is the external input, $d(t) \in \mathcal{L}_{\infty}$, and $f: \mathbb{R}^{n+m} \rightarrow \mathbb{R}^{n}$ is a locally Lipschitz (or 
Hölder) continuous function, $f(0,0)=0$. In some cases we will equip the system (1) with an output $y \in \mathbb{R}^{p}$ :

$$
y=h(x),
$$

where $h: \mathbb{R}^{n} \rightarrow \mathbb{R}^{p}$ is a continuous function. For an initial condition $x_{0} \in \mathbb{R}^{n}$ and input $d \in \mathcal{L}_{\infty}$, define the corresponding solutions by $x\left(t, x_{0}, d\right)$ for any $t \geq 0$ for which the solution exists.

\section{A. Stability properties}

In this work we will be interested in the following stability properties [8].

Definition 1. The system (1) is called input-to-state practically stable (ISpS), if for any input $d \in \mathcal{L}_{\infty}$ and any $x_{0} \in \mathbb{R}^{n}$ there are some functions $\beta \in \mathcal{K} \mathcal{L}, \gamma \in \mathcal{K}$ and $c \geq 0$ such that

$$
\left\|x\left(t, x_{0}, d\right)\right\| \leq \beta\left(\left\|x_{0}\right\|, t\right)+\gamma\left(\|d\|_{[0, t)}\right)+c \quad \forall t \geq 0 .
$$

The function $\gamma$ is called nonlinear asymptotic gain. The system is called ISS if $c=0$.

Definition 2. The system (1) is called iISS, if there are some functions $\alpha \in \mathcal{K}_{\infty}, \gamma \in \mathcal{K}$ and $\beta \in \mathcal{K} \mathcal{L}$ such that for any $x_{0} \in \mathbb{R}^{n}$ and $d \in \mathcal{L}_{\infty}$ the estimate holds:

$$
\alpha\left(\left\|x\left(t, x_{0}, d\right)\right\|\right) \leq \beta\left(\left\|x_{0}\right\|, t\right)+\int_{0}^{t} \gamma(\|d(s)\|) d s \quad \forall t \geq 0 .
$$

These properties have the following Lyapunov function characterizations.

Definition 3. A smooth function $V: \mathbb{R}^{n} \rightarrow \mathbb{R}_{+}$is called ISpS Lyapunov function for the system (1) if for all $x \in \mathbb{R}^{n}$, $d \in \mathbb{R}^{m}$ and some $r \geq 0, \alpha_{1}, \alpha_{2}, \alpha_{3} \in \mathcal{K}_{\infty}$ and $\theta \in \mathcal{K}$ :

$$
\begin{aligned}
\alpha_{1}(\|x\|) & \leq V(x) \leq \alpha_{2}(\|x\|), \\
D V(x) f(x, d) & \leq r+\theta(\|d\|)-\alpha_{3}(\|x\|) .
\end{aligned}
$$

Such a function $V$ is called ISS Lyapunov function if $r=0$, and it is ilSS Lyapunov function if instead $\alpha_{3}: \mathbb{R}_{+} \rightarrow \mathbb{R}_{+}$is a positive definite function.

Note that an ISS Lyapunov function can also satisfy the following equivalent condition for some $\chi \in \mathcal{K}$ :

$$
\|d\| \leq \chi(\|x\|) \Rightarrow D V(x) f(x, d) \leq-\alpha_{3}(\|x\|) .
$$

Theorem 1. The system (1) is ISS (ISpS, iISS) iff it admits an ISS (ISpS, iISS) Lyapunov function.

Note that, if the system (1) is ISS, then it is also iISS.

\section{B. Weighted homogeneity}

Following [19], for any strictly positive numbers $\lambda$ and $r_{i}$, $i=\overline{1, n}$ called weights, one can define:

- the vector of weights $\mathbf{r}=\left(r_{1}, \ldots, r_{n}\right)^{T}, r_{\max }=$ $\max _{1 \leq j \leq n} r_{j}$ and $r_{\min }=\min _{1 \leq j \leq n} r_{j}$

- the dilation matrix $\Lambda_{r}=\operatorname{diag}\left\{\lambda^{r_{i}}\right\}_{i=1}^{n}$, note that for any $x \in \mathbb{R}^{n}$ we have $\Lambda_{r} x=$ $\left(\lambda^{r_{1}} x_{1}, \ldots, \lambda^{r_{i}} x_{i}, \ldots, \lambda^{r_{n}} x_{n}\right)^{T}$;
- the $r$-homogeneous norm $\|x\|_{r}=\left(\sum_{i=1}^{n}\left|x_{i}\right|^{\frac{\rho}{r_{i}}}\right)^{\frac{1}{\rho}}$ for any $x \in \mathbb{R}^{n}$ and some $\rho>0$;

- the unit sphere in the homogeneous norm $S_{r}=\{x \in$ $\left.\mathbb{R}^{n}:\|x\|_{r}=1\right\}$.

Definition 4. A function $g: \mathbb{R}^{n} \rightarrow \mathbb{R}$ is $\mathbf{r}$-homogeneous with degree $\mu \in \mathbb{R}$ if for all $x \in \mathbb{R}^{n}$ we have:

$$
\lambda^{-\mu} g\left(\Lambda_{r} x\right)=g(x) .
$$

A vector field $f: \mathbb{R}^{n} \rightarrow \mathbb{R}^{n}$ is $\mathbf{r}$-homogeneous with degree $\nu \in \mathbb{R}$, with $\nu \geq-r_{\min }$ if for all $x \in \mathbb{R}^{n}$ we have:

$$
\lambda^{-\nu} \Lambda_{r}^{-1} f\left(\Lambda_{r} x\right)=f(x),
$$

which is equivalent for $i$-th component of $f$ being a $\mathbf{r}-$ homogeneous function of degree $r_{i}+\nu$.

The system (1) with $d=0$ is $\mathbf{r}$-homogeneous of degree $\nu$ if the vector field $f$ is $\mathbf{r}$-homogeneous of degree $\nu$.

Theorem 2. [13] For the system (1) with $d=0$ and $\mathbf{r}$ homogeneous and continuous function $f$ the following properties are equivalent:

- the system (1) is (locally) asymptotically stable;

- there exists a continuously differentiable $\mathbf{r}-$ homogeneous Lyapunov function $V: \mathbb{R}^{n} \rightarrow \mathbb{R}_{+}$such that

$$
\begin{gathered}
\alpha_{1}(\|x\|) \leq V(x) \leq \alpha_{2}(\|x\|), D V(x) f(x, 0) \leq-\alpha(\|x\|), \\
\lambda^{-\mu} V\left(\Lambda_{r} x\right)=V(x), \mu>r_{\max },
\end{gathered}
$$

for all $x \in \mathbb{R}^{n}$, for some $\alpha_{1}, \alpha_{2} \in \mathcal{K}_{\infty}$ and $\alpha \in \mathcal{K}$.

The $\mathbf{r}$-homogeneity concept presented in Definition 4 is introduced for some $\mathbf{r}$ and all $\lambda>0$. Restricting the set of admissible values for $\lambda$ we can introduce local homogeneity [15], [16].

Definition 5. A function $g: \mathbb{R}^{n} \rightarrow \mathbb{R}$ is $\left(\mathbf{r}_{0}, \lambda_{0}, g_{0}\right)-$ homogeneous with degree $\nu_{0} \in \mathbb{R}$ ( $g_{0}$ is a $\mathbf{r}_{0}$-homogeneous function and $\lambda_{0} \in \mathbb{R}_{+} \cup\{+\infty\}$ ) if for all $x \in S_{r_{0}}$ we have:

$$
\lim _{\lambda \rightarrow \lambda_{0}}\left(\lambda^{-\nu_{0}} g\left(\Lambda_{r_{0}} x\right)-g_{0}(x)\right)=0,
$$

uniformly on $S_{r_{0}}$ for $\lambda_{0} \in\{0,+\infty\}$.

A vector field $f: \mathbb{R}^{n} \rightarrow \mathbb{R}^{n}$ is $\left(\mathbf{r}_{0}, \lambda_{0}, f_{0}\right)$-homogeneous with degree $\mu_{0} \geq-r_{0 \text { min }}$ ( $f_{0}$ is a $\mathbf{r}_{0}$-homogeneous vector field and $\lambda_{0} \in \mathbb{R}_{+} \cup\{+\infty\}$ ) if for all $x \in S_{r_{0}}$ we have:

$$
\lim _{\lambda \rightarrow \lambda_{0}}\left(\lambda^{-\mu_{0}} \Lambda_{r_{0}}^{-1} f\left(\Lambda_{r_{0}} x\right)-f_{0}(x)\right)=0,
$$

uniformly on $S_{r_{0}}$ for $\lambda_{0} \in\{0,+\infty\}$.

The system (1) for $d=0$ is $\left(\mathbf{r}_{0}, \lambda_{0}, f_{0}\right)$-homogeneous with degree $\mu_{0} \in \mathbb{R}$ if the vector field $f$ is $\left(\mathbf{r}_{0}, \lambda_{0}, f_{0}\right)$ homogeneous with degree $\mu_{0}$.

The coefficients $r_{0 i}>0, i \in \overline{1, n}$ are called weights, $\nu_{0}$ (respectively $\mu_{0}$ ) is the degree of homogeneity (it may depend on $\lambda_{0}$ ) and $g_{0}$ (respectively $f_{0}$ ) is the approximating function of $g$ (respectively $f$ ) at $\lambda_{0}$.

Theorem 3. [20], [13] Let the system (1) with $d=0$ be $\left(\mathbf{r}, 0, f_{0}\right)$-homogeneous with a continuous $f_{0}: \mathbb{R}^{n} \rightarrow \mathbb{R}^{n}$. If the system $\dot{x}=f_{0}(x)$ is (locally) asymptotically stable, then the system (1) is also locally asymptotically stable. 
Theorem 4. [15] Let the system (1) with $d=0$ be $\left(\mathbf{r},+\infty, f_{\infty}\right)$-homogeneous with a continuous $f_{\infty}: \mathbb{R}^{n} \rightarrow \mathbb{R}^{n}$. If the system $\dot{x}=f_{\infty}(x)$ is (globally) asymptotically stable, then there exists a compact invariant set $X_{\infty} \subset \mathbb{R}^{n}$ containing the origin such that the system (1) is globally asymptotically stable with respect to the set $X_{\infty}$.

Lemma 1. Let a function $g: \mathbb{R}^{n} \rightarrow \mathbb{R}$ be $\left(\mathbf{r},+\infty, g_{\infty}\right)-$ homogeneous with degree $\nu, g$ and $g_{\infty}$ be locally Lipschitz continuous functions, then for all $x \in \mathbb{R}^{n}$

$$
\left|g(x)-g_{\infty}(x)\right| \leq \omega\left(\|x\|_{r}\right), \omega(s)=k\left\{\begin{array}{ll}
s^{w_{\min }} & \text { if } s \leq 1 \\
s^{w_{\max }} & \text { if } s>1
\end{array},\right.
$$

where $k>0$ and $0 \leq w_{\min } \leq \nu, 0 \leq w_{\max }<\nu$.

Proof: By definition for any $x \in \mathbb{R}^{n}$ there are $y \in S_{r}$ and $\lambda=\|x\|_{r}$ such that $x=\Lambda_{r} y$, then $\left|g(x)-g_{\infty}(x)\right|=$ $\left|g\left(\Lambda_{r} y\right)-g_{\infty}\left(\Lambda_{r} y\right)\right|$. For any $y \in S_{r}$ consider

$$
\left|\lambda^{-\nu} g\left(\Lambda_{r} y\right)-g_{\infty}(y)\right|=\lambda^{-\nu}\left|g\left(\Lambda_{r} y\right)-g_{\infty}\left(\Lambda_{r} y\right)\right| .
$$

For $\lambda \rightarrow+\infty$ the left hand side of the relation above converges to zero, therefore there is $\bar{k}>0$ such that $\left|g\left(\Lambda_{r} y\right)-g_{\infty}\left(\Lambda_{r} y\right)\right| \leq \bar{k} \lambda^{w_{\max }}$ with $w_{\max }<\nu$ for all $\lambda>1$. Next, $\left|g\left(\Lambda_{r} y\right)-g_{\infty}\left(\Lambda_{r} y\right)\right| \leq \underline{k} \lambda^{w_{\min }}$ for $\lambda \leq 1$ with some $\underline{k}>0$ and $w_{\min } \geq 0$ due to Lipschitz continuity of $g$ and $g_{\infty}$, additionally $w_{\min } \leq \nu$ (by a proper choice of $\underline{k}$ ), then $k=\max \{\underline{k}, \bar{k}\}$.

Clearly, it is always possible to select the powers in a way that $0 \leq w_{\min } \leq w_{\max }<\nu$.

Lemma 2. Let a vector field $f: \mathbb{R}^{n} \rightarrow \mathbb{R}^{n}$ be $\left(\mathbf{r},+\infty, f_{\infty}\right)-$ homogeneous with degree $\nu, f$ and $f_{\infty}$ be locally Lipschitz continuous, then for all $x \in \mathbb{R}^{n}$

$$
\left\|f(x)-f_{\infty}(x)\right\| \leq \omega\left(\|x\|_{r}\right), \omega(s)=\left\{\begin{array}{ll}
k s^{w_{\min }} & \text { if } s \leq 1 \\
k s^{w_{\max }} & \text { if } s>1
\end{array},\right.
$$

where $k>0$ and $0 \leq w_{\min } \leq w_{\max }<r_{\max }+\nu$.

Proof: In this case each $f_{i}$ is $\left(\mathbf{r},+\infty, f_{\infty i}\right)$-homogeneous with degree $\nu+r_{i}$ for all $1 \leq i \leq n$, and the result follows Lemma 1.

\section{ROBUSTNESS OF HOMOGENEOUS SYSTEMS}

The ISS property of a $\mathbf{r}$-homogeneous system (1) with degree $\nu>1$ has been investigated in [18], the ISS property of a $\mathbf{r}$-homogeneous system of the form

$$
\dot{x}=f_{0}(x)+G_{0}(x) d
$$

for any admissible degree $\nu \geq-r_{\min }$ (with homogeneous $f_{0}$ and $G_{0}$ ) has been studied in [17]. In this work we would like to propose the conditions of ISS and iISS properties for a $\mathbf{r}$-homogeneous system (1) with any $\nu \geq-r_{\min }$.

Define $\tilde{f}(x, d)=\left[f(x, d)^{T} 0_{m}\right]^{T} \in \mathbb{R}^{\bar{n}+m}$, it is an extended auxiliary vector field for the system (1), where $0_{m}$ is the zero vector of dimension $m$.

Theorem 5. Let the vector field $\tilde{f}$ be homogeneous with the weights $\mathbf{r}=\left[r_{1}, \ldots, r_{n}\right]>0, \tilde{\mathbf{r}}=\left[\tilde{r}_{1}, \ldots, \tilde{r}_{m}\right] \geq 0$ with a degree $\nu \geq-r_{\min }$, i.e. $f\left(\Lambda_{r} x, \Lambda_{\tilde{r}} d\right)=\lambda^{\nu} \Lambda_{r} f(x, d)$. Assume that the system (1) is globally asymptotically stable for $d=0$, then the system (1) is

$\begin{array}{ll}\text { ISS } & \text { if } \tilde{r}_{\min }>0 \text {, where } \tilde{r}_{\min }=\min _{1 \leq j \leq m} \tilde{r}_{j} ; \\ \text { iISS } & \text { if } \tilde{r}_{\min }=0 \text { and } \nu \leq 0 .\end{array}$

Proof: Under the introduced conditions $f\left(\Lambda_{r} x, 0\right)=$ $\lambda^{\nu} \Lambda_{r} f(x, 0)$ and the system $\dot{x}=f(x, 0)$ is globally asymptotically stable, therefore by Theorem 2 there exists a continuously differentiable, positive definite and radially unbounded Lyapunov function $V: \mathbb{R}^{n} \rightarrow \mathbb{R}_{+}$such that $V\left(\Lambda_{r} x\right)=\lambda^{\mu} V(x)$ for any $\mu>r_{\max }$ and

$$
D V(y) f(y, 0) \leq-a,\left\|\frac{\partial V(y)}{\partial y}\right\| \leq b \quad \forall y \in S_{r},
$$

where $a>0, b>0$. Note that by definition in this case $\nu+\mu>0$. Due to homogeneity of $\tilde{f}$ and continuity of $f$ with respect to $d$ we have

$$
\begin{array}{r}
\|f(y, d)-f(y, 0)\| \leq \sigma(\|d\|) \quad \forall y \in S_{r}, \\
\sigma(s)=c \begin{cases}s^{\varrho_{\min }} & \text { if } s \leq 1 \\
s^{\varrho_{\max }} & \text { if } s>1\end{cases}
\end{array}
$$

for some $c>0$ and $\varrho_{\max } \geq \varrho_{\min }>0$.

Below we will use the coordinate transformation $x=\Lambda_{|x|} y$, where $\Lambda_{|x|}=\left.\Lambda_{r}\right|_{\lambda=\|x\|_{r}}$, which connects any $x \in \mathbb{R}^{n} \backslash\{0\}$ with the corresponding point $y \in S_{r}$. For the input $d$ we will use the transformation $d=\tilde{\Lambda}_{|x|} \tilde{d}$, where $\tilde{d} \in \mathbb{R}^{m}$ and $\tilde{\Lambda}_{|x|}=\left.\Lambda_{\tilde{r}}\right|_{\lambda=\|x\|_{r}}$, then

$$
\|\tilde{d}\| \leq \rho\left(\|x\|_{r}\right)\|d\|, \rho(s)=\left\{\begin{array}{ll}
s^{-\tilde{r}_{\max }} & \text { if } s \leq 1 \\
s^{-\tilde{r}_{\min }} & \text { if } s>1
\end{array},\right.
$$

where $\tilde{r}_{\max }=\max _{1 \leq j \leq m} \tilde{r}_{j}$. Now let us consider the time derivative of the Lyapunov function $V$ computed for the system (1) for all $x \in \mathbb{R}^{n}$ and $d \in \mathbb{R}^{m}$ :

$$
\begin{aligned}
D V(x) f(x, d)= & \|x\|_{r}^{\nu+\mu} \frac{\partial V(y)}{\partial y} f(y, \tilde{d}) \\
= & \|x\|_{r}^{\nu+\mu} \frac{\partial V(y)}{\partial y} f(y, 0) \\
& +\|x\|_{r}^{\nu+\mu} \frac{\partial V(y)}{\partial y}[f(y, \tilde{d})-f(y, 0)] \\
\leq & -a\|x\|_{r}^{\nu+\mu}+b\|x\|_{r}^{\nu+\mu} \sigma(\|\tilde{d}\|) \\
\leq & -a\|x\|_{r}^{\nu+\mu}+b\|x\|_{r}^{\nu+\mu} \gamma\left(\|x\|_{r}\right) \sigma(\|d\|)
\end{aligned}
$$

where

$$
\gamma(s)=\left\{\begin{array}{ll}
s^{-\tilde{r}_{\max } \varrho_{\min }} & \text { if } s \leq 1 \\
s^{-\tilde{r}_{\min } \varrho_{\max }} & \text { if } s>1
\end{array} .\right.
$$

Therefore if $-\tilde{r}_{\min } \varrho_{\max }<0$, which is equivalent to $\tilde{r}_{\min }>0$, then $\gamma(s)^{-1} \in \mathcal{K}_{\infty}$ and for $\|d\| \leq \sigma^{-1}\left[a /(2 b) \gamma\left(\|x\|_{r}\right)^{-1}\right]$ we have $D V(x) f(x, d) \leq-0.5 a\|x\|_{r}^{\nu+\mu}$ that implies ISS by Theorem 1. If $\tilde{r}_{\max } \varrho_{\min }-\mu \leq \nu \leq \tilde{r}_{\min } \varrho_{\max }$, then

$$
\|x\|_{r}^{\nu+\mu} \gamma\left(\|x\|_{r}\right) \leq v\left(\|x\|_{r}\right), \quad v(s)= \begin{cases}1 & \text { if } s \leq 1 \\ s^{\mu} & \text { if } s>1\end{cases}
$$

and for the Lyapunov function $W(x)=\ln [1+V(x)]$ (it is continuously differentiable, positive definite and radially 
unbounded) we obtain

$$
\begin{aligned}
D W(x) f(x, d) & \leq \frac{-a\|x\|_{r}^{\nu+\mu}+b v\left(\|x\|_{r}\right) \sigma(\|d\|)}{1+V(x)} \\
& \leq-a \frac{\|x\|_{r}^{\nu+\mu}}{1+V(x)}+\tilde{b} \sigma(\|d\|), \tilde{b}>0
\end{aligned}
$$

which implies iISS for (1). Since $\mu>r_{\max }$ can be chosen arbitrary, then we may assume that the inequality $\tilde{r}_{\max } \varrho_{\min }-$ $\mu \leq \nu$ is always satisfied. Since for $\tilde{r}_{\text {min }}>0$ the system is ISS (and iISS by the definition), the only interesting admissible value is $\tilde{r}_{\min }=0$, then $\nu$ has to be non positive.

As we can conclude from this result, for the homogeneous system (1) its robustness (ISS or iISS property) is a function of its degree of homogeneity.

Corollary 1. Let a locally Lipschitz continuous function $f_{0}: \mathbb{R}^{n} \rightarrow \mathbb{R}^{n}$ be $\mathbf{r}$-homogeneous with a degree $\nu$ and asymptotically stable.

If $f(x, d)=f_{0}(x)+d$, i.e. $d$ is an additive disturbance, then the system (1) is ISS for $\nu>-r_{\min }$, and iISS for $\nu=-r_{\min }$.

If $f(x, d)=f_{0}(x+d)$, i.e. $d$ is a measurement noise, then the system (1) is always ISS.

Proof: Take $\tilde{\mathbf{r}}=\mathbf{r}+\nu$ and $\tilde{\mathbf{r}}=\mathbf{r}$ for the additive disturbance and measurement noise cases respectively.

Thus to verify robustness of a system with respect to an external input it is enough to establish its asymptotic stability for the case $d=0$ and compute its degree of homogeneity performing some algebraic operations, which is a big advantage of the homogeneity approach. However, the sole homogeneity of $\tilde{f}$ is not enough to claim iISS (ISS), and the case $\tilde{r}_{\min }=0$ with $\nu>0$ is the only exclusion as in the following example for $\tilde{\mathbf{r}}=0$ and $\mathbf{r}=1$ :

$$
\dot{x}=(d-1) x^{\alpha}, \alpha>1 .
$$

The asymptotically stable system (1) for $d=0$ is finitetime stable if it is homogeneous with negative degree [21], [22]. Interestingly to note that the finite-time stability and iISS have a similar restriction on the degree of homogeneity (it has to be negative or non positive for iISS), thus the finite-time stability of a homogeneous system implies iISS.

Corollary 2. Let the vector field $\tilde{f}$ be homogeneous with the weights $\mathbf{r}=\left[r_{1}, \ldots, r_{n}\right]>0, \tilde{\mathbf{r}}=\left[\tilde{r}_{1}, \ldots, \tilde{r}_{m}\right] \geq 0$ with a degree $0>\nu \geq-r_{\min }$ and asymptotically stable for $d=0$, then (1) is iISS.

Theorem 5 also provides a quantitative estimate on the asymptotic gain of (1) in the ISS case.

Corollary 3. Let the system (1) be ISS under conditions of Theorem 5 and $\nu+\mu>\tilde{r}_{\min } \varrho_{\max }$, then its asymptotic gain $\gamma$ admits the estimate:

$$
\gamma(s) \leq \ell\left\{\begin{array}{ll}
s^{\frac{\varrho_{\min }}{\nu+\mu}} & \text { if } s \leq 1 \\
s^{\tilde{r}_{\min }^{-1}} & \text { if } s>1
\end{array}, \ell>0\right.
$$

The case $\nu+\mu \leq \tilde{r}_{\min } \varrho_{\max }$ has no practical importance and will not be treated here.
Proof: Since in the ISS case $\tilde{r}_{\min }>0$ and $\|x\|_{r}^{\nu+\mu} \gamma\left(\|x\|_{r}\right) \leq 1+\|x\|_{r}^{\nu+\mu-\tilde{r}_{\min } \varrho_{\max }}$, then following the proof of Theorem 5 we obtain:

$D V(x) f(x, d) \leq-a\|x\|_{r}^{\nu+\mu}+b\left[1+\|x\|_{r}^{\nu+\mu-\tilde{r}_{\min } \varrho_{\max }}\right] \sigma(\|d\|)$.

From Young's inequality we get:

$$
s^{\nu+\mu-\tilde{r}_{\min } \varrho_{\max }} v \leq \frac{a}{2 b} s^{\nu+\mu}+\pi_{1}^{-1} v^{\pi_{1}} \varphi
$$

where $\pi_{1}=\frac{\nu+\mu}{\tilde{r}_{\min } \varrho_{\max }}>1$ and $\varphi=\left[\frac{a}{2 b} \frac{\pi_{1}}{\pi_{1}-1}\right]^{1-\pi_{1}}$. Since

$$
\begin{aligned}
b\left[\sigma(s)+\pi_{1}^{-1} \varphi \sigma(s)^{\pi_{1}}\right] & \leq \psi(s) \\
& =z \begin{cases}s^{\varrho_{\min }} & \text { if } s \leq 1 \\
s^{\varrho_{\max } \pi_{1}} & \text { if } s>1\end{cases}
\end{aligned}
$$

for $z=2 b \max \left\{1, \pi_{1}^{-1} \varphi\right\} \max \left\{c, c^{\pi_{1}}\right\}$, then

$$
D V(x) f(x, d) \leq-0.5 a\|x\|_{r}^{\nu+\mu}+\psi(\|d\|) .
$$

Therefore $D V(x) f(x, d) \leq-q V(x)^{\frac{\nu+\mu}{\mu}}+\psi(\|d\|)$ for some $q>0$, and from the estimate $\|x\|_{r}^{\nu+\mu} \leq V(x)^{\frac{\nu+\mu}{\mu}} \leq$ $2 q^{-1} \psi(\|d\|)$ we obtain the proposed expression for the asymptotic gain.

The case $\tilde{r}_{\min }=0$ is critical for Theorem 5 , it is possible that the system (1) is ISS while $\tilde{r}_{\text {min }}=0$ as it is shown in the following example:

$$
\begin{aligned}
& \dot{x}_{1}=-x_{1}^{3}+x_{2}^{2} d_{1}, \\
& \dot{x}_{2}=-x_{2}^{7 / 3}+\left|x_{1}\right|^{1 / 2} d_{2},
\end{aligned}
$$

where $\mathbf{r}=\left[\begin{array}{ll}1 & 1.5\end{array}\right], \tilde{\mathbf{r}}=\left[\begin{array}{ll}0 & 3\end{array}\right], \nu=2$ and its ISS Lyapunov function is $V(x)=0.5 x_{1}^{2}+0.5 x_{2}^{2}$.

The conditions of Theorem 5 can be technically relaxed skipping homogeneity of $\tilde{f}$ (homogeneity with respect to $d$ ). It is worth stressing that homogeneity of $\tilde{f}$ is not a restrictive condition since $d$ is an external input, and we can modify dimension or introduce nonlinear change of coordinates for $d$.

Theorem 6. Assume that the system (1) is globally asymptotically stable for $d=0$ and $\mathbf{r}$-homogeneous with a degree $\nu \geq-r_{\min }$, i.e. $f\left(\Lambda_{r} x, 0\right)=\lambda^{\nu} \Lambda_{r} f(x, 0)$. Let also for all $x \in \mathbb{R}^{n}$ and $d \in \mathbb{R}^{m}$

$$
\begin{aligned}
& \|f(x, d)-f(x, 0)\| \leq \theta\left(\|x\|_{r}\right) \psi(\|d\|)+\phi(\|d\|), \\
& \theta(s)=\left\{\begin{array}{ll}
s^{\vartheta_{\min }} & \text { if } s \leq 1 \\
s^{\vartheta_{\max }} & \text { if } s>1
\end{array}, \vartheta_{\min } \geq 0, \vartheta_{\max } \in \mathbb{R}\right.
\end{aligned}
$$

for some $\psi, \phi \in \mathcal{K}$. Then the system (1) is

$$
\begin{array}{ll}
\text { ISS } & \text { if } \nu>\vartheta_{\max }-r_{\min } \\
\text { iISS } & \text { if } \nu=\vartheta_{\max }-r_{\min } \leq 0 .
\end{array}
$$

Proof: Under introduced conditions, by Theorem 2 there exists a continuously differentiable, positive definite and radially unbounded Lyapunov function $V: \mathbb{R}^{n} \rightarrow \mathbb{R}_{+}$such that $V\left(\Lambda_{r} x\right)=\lambda^{\mu} V(x)$ for any $\mu>r_{\max }$ with $\nu+\mu>0$, and the inequalities (4) are satisfied for $a>0, b>0$. Consider the time derivative of the Lyapunov function $V$ computed for the system (1) for all $x \in \mathbb{R}^{n}$ and $d \in \mathbb{R}^{m}$ using the 
coordinate transformation $x=\Lambda_{|x|} y$ with $\Lambda_{|x|}=\left.\Lambda_{r}\right|_{\lambda=\|x\|_{r}}$ $\left(\|x\|_{r}=\left\|\Lambda_{|x|} y\right\|_{r}\right):$

$$
\begin{aligned}
& D V(x) f(x, d)=\frac{\partial V(x)}{\partial x} f(x, 0)+\frac{\partial V(x)}{\partial x}[f(x, d)-f(x, 0)] \\
&=\quad\|x\|_{r}^{\nu+\mu} \frac{\partial V(y)}{\partial y} f(y, 0) \\
& \quad+\|x\|_{r}^{\mu} \frac{\partial V(y)}{\partial y} \Lambda_{|x|}^{-1}\left[f\left(\Lambda_{|x|} y, d\right)-f\left(\Lambda_{|x|} y, 0\right)\right] \\
& \leq \quad-a\|x\|_{r}^{\nu+\mu}+b\|x\|_{r}^{\mu}\left\|\Lambda_{|x|}^{-1}\right\|\left[\theta\left(\left\|\Lambda_{|x|} y\right\|_{r}\right) \psi(\|d\|)\right. \\
&\quad+\phi(\|d\|)] \\
& \leq \quad-a\|x\|_{r}^{\nu+\mu}+b\|x\|_{r}^{\nu+\mu}\left[\gamma_{1}\left(\|x\|_{r}\right) \psi(\|d\|)\right. \\
&\left.+\gamma_{2}\left(\|x\|_{r}\right) \phi(\|d\|)\right],
\end{aligned}
$$

where

$$
\begin{aligned}
& \gamma_{1}(s)=\left\{\begin{array}{ll}
s^{\vartheta_{\min }-r_{\max }-\nu} & \text { if } s \leq 1 \\
s^{\vartheta_{\max }-r_{\min }-\nu} & \text { if } s>1
\end{array},\right. \\
& \gamma_{2}(s)=\left\{\begin{array}{ll}
s^{-r_{\max }-\nu} & \text { if } s \leq 1 \\
s^{-r_{\min }-\nu} & \text { if } s>1
\end{array} .\right.
\end{aligned}
$$

Therefore if $\vartheta_{\max }-r_{\min }-\nu<0$, then $\gamma_{1}(s)^{-1}$ and $\gamma_{2}(s)^{-1}$ are in $\mathcal{K}_{\infty}$, and for $\|d\| \leq$ $\min \left\{\psi^{-1}\left[a /(4 b) \gamma_{1}\left(\|x\|_{r}\right)^{-1}\right], \phi^{-1}\left[a /(4 b) \gamma_{2}\left(\|x\|_{r}\right)^{-1}\right]\right\}$

we have $D V(x) f(x, d) \leq-0.5 a\|x\|_{r}^{\nu+\mu}$ that, by Theorem 1 , implies the ISS property. If $\vartheta_{\max }-r_{\min }-\nu=0$, $\nu \leq 0$ and $\vartheta_{\min }-r_{\max }+\mu \geq 0$ (the latter is true since $\left.\mu>r_{\max }\right)$, then $\|x\|_{r}^{\nu+\mu} \gamma_{1}\left(\|x\|_{r}\right) \leq v\left(\|x\|_{r}\right)$ and $\|x\|_{r}^{\nu+\mu} \gamma_{2}\left(\|x\|_{r}\right) \leq v\left(\|x\|_{r}\right)$, where $v(s)$ is defined at (6) and for the Lyapunov function $W(x)=\ln [1+V(x)]$ we obtain (7) for $\sigma(s)=2 \max \{\psi(s), \phi(s)\}$, which implies iISS for (1).

The result of Theorem 6 can be applied for a larger class of systems, which are not necessarily homogeneous (the function $\tilde{f}$ may be non homogeneous). For example, to the system (3) with non homogeneous $G_{0}$ (the result of [17] cannot be used in this case):

$$
\begin{aligned}
& \dot{x}_{1}=-x_{1}+x_{2} d_{1} /\left(1+\left|x_{2}\right|\right), \\
& \dot{x}_{2}=-x_{2}+x_{1}^{1 / 3} d_{2},
\end{aligned}
$$

where $\mathbf{r}=\left[\begin{array}{ll}1 & 1\end{array}\right]$ and $\nu=0$ for $d=0, \vartheta_{\min }=\vartheta_{\max }=1 / 3$. However, the conditions obtained in Theorem 6 also do not work for the critical case example (8), where $\vartheta_{\min }=0.5$, $\vartheta_{\max }=3$ and the equality $\nu=\vartheta_{\max }-r_{\min }$ is satisfied. A reason of that is hidden in the conservatism of the function $\theta$ computation. Another explanation of this fact is that, in the case $\tilde{r}_{\min }=0$ the system (1) may not admit a $\mathbf{r}-$ homogeneous ISS Lyapunov function (both theorems 5 and 6 are based on an ISS Lyapunov function of that type), see also the case of Proposition 1 below, where this hypothesis is proven for the case $\tilde{\mathbf{r}}=0_{m}$.

Proposition 1. Considering $d$ as a constant, let the vector field $f$ be $\mathbf{r}$-homogeneous with a degree $\nu \geq-r_{\min }$ independent of d, i.e. $f\left(\Lambda_{r} x, d\right)=\lambda^{\nu} \Lambda_{r} f(x, d)$ for any $x \in \mathbb{R}^{n}$ and $d \in$ $\mathbb{R}^{m}$. Assume that the system (1) is ISS, then there is no $\mathbf{r}-$ homogeneous ISS Lyapunov functions for (1).
Proof: By definition, an ISS Lyapunov function for the system (1) is a Lyapunov function for $d=0$. Take any $\mathbf{r}$ homogeneous Lyapunov function for the system (1) with $d=$ 0 , say $V(x)$, then for any $x \in \mathbb{R}^{n}, d \in \mathbb{R}^{m}$ and $y \in S_{r}$ we obtain

$$
D V(x) f(x, d)=\|x\|_{r}^{\nu+\mu} c_{1}(y)+\|x\|_{r}^{\nu+\mu} c_{2}(y, d),
$$

where $c_{1}(y)=\frac{\partial V(y)}{\partial y} f(y, 0)<0$ and $c_{2}(y, d)=$ $\frac{\partial V(y)}{\partial y}[f(y, d)-f(y, 0)] \leq \sigma(\|d\|)$ for some $\sigma \in \mathcal{K}$. Therefore, $V$ cannot be an ISS Lyapunov function.

Note that an iISS Lyapunov function cannot be homogeneous since $\alpha_{3}$ is a bounded positive definite function in this case (if the system is not ISS, see Definition 3), while from consideration above $\alpha_{3}$ is proportional to $\|x\|_{r}^{\nu+\mu}$ for a homogeneous function $V$. However, the case of Proposition 1 still can be useful for the iISS property.

Theorem 7. Let the vector field $f$ be $\mathbf{r}$-homogeneous with a degree $0 \geq \nu \geq-r_{\min }$ considering $d$ as a constant, i.e. $f\left(\Lambda_{r} x, d\right)=\lambda^{\nu} \Lambda_{r} f(x, d)$ for any $x \in \mathbb{R}^{n}$ and $d \in \mathbb{R}^{m}$. Then the system (1) is globally asymptotically stable for $d=0$ iff it is iISS.

Proof: Under introduced conditions $f\left(\Lambda_{r} x, 0\right)=$ $\lambda^{\nu} \Lambda_{r} f(x, 0)$ and the system $\dot{x}=f(x, 0)$ is globally asymptotically stable, therefore by Theorem 2 there exists a continuously differentiable, positive definite and radially unbounded Lyapunov function $V: \mathbb{R}^{n} \rightarrow \mathbb{R}_{+}$such that $V\left(\Lambda_{r} x\right)=\lambda^{\mu} V(x)$ for any $\mu>r_{\max }(\nu+\mu>0)$, and the inequalities (4) are satisfied for $a>0, b>0$. Due to continuity of $f$ with respect to $d$ we have for some $\sigma \in \mathcal{K}$

$$
\|f(y, d)-f(y, 0)\| \leq \sigma(\|d\|) \quad \forall y \in S_{r} .
$$

Now let us consider the time derivative of the Lyapunov function $V$ computed for the system (1) for all $x \in \mathbb{R}^{n}$ and $d \in \mathbb{R}^{m}$ using the coordinate transformation $x=\Lambda_{|x|} y$ with $\Lambda_{|x|}=\left.\Lambda_{r}\right|_{\lambda=\|x\|_{r}}$ :

$$
\begin{aligned}
D V(x) f(x, d)= & \|x\|_{r}^{\nu+\mu} \frac{\partial V(y)}{\partial y} f(y, 0) \\
& +\|x\|_{r}^{\nu+\mu} \frac{\partial V(y)}{\partial y}[f(y, d)-f(y, 0)] \\
\leq & -a\|x\|_{r}^{\nu+\mu}+b\|x\|_{r}^{\nu+\mu} \sigma(\|d\|) .
\end{aligned}
$$

Therefore, if $\nu \leq 0$, then $\|x\|_{r}^{\nu+\mu} \leq v\left(\|x\|_{r}\right)$, where $v(s)$ is defined in (6), and for the Lyapunov function $W(x)=$ $\ln [1+V(x)]$ we obtain (7) that according to Theorem 1 implies iISS for (1).

This result can be applied, for example, to "bilinear" systems:

$$
\dot{x}=f_{0}(x)+\sum_{i=1}^{m} f_{i}\left(x d_{i}\right),
$$

where all $f_{i}, i=0, \ldots, m$ are $\mathbf{r}-$ homogeneous functions of the same degree with respect to $x, f_{i}(0)=0$ (a simplest example is $f_{i}(x)=A_{i} x$, where $\left.A_{i} \in \mathbb{R}^{n \times n}\right)$. According to Theorem 7 , if in (9) the system $\dot{x}=f_{0}(x)$ is asymptotically stable and the homogeneity degree is non-positive, then the system is iISS. 
To finish comparison of theorems 5 and 6 note that the conditions of Theorem 6 may be more restrictive than in Theorem 5 , as it can be demonstrated in the following example:

$$
\begin{aligned}
& \dot{x}_{1}=-x_{1}^{3}+x_{2}^{1 / 3} d_{1}, \\
& \dot{x}_{2}=-x_{2}^{5 / 3}+x_{1}^{3} d_{2},
\end{aligned}
$$

where $\mathbf{r}=\left[\begin{array}{ll}1 & 3\end{array}\right], \tilde{\mathbf{r}}=\left[\begin{array}{ll}2 & 2\end{array}\right], \nu=2$ and it is ISS by Theorem 5 (it also has a homogeneous ISS Lyapunov function $\left.V(x)=x_{1}^{6} / 6+x_{2}^{2} / 2\right)$, but Theorem 6 does not work since $\vartheta_{\text {min }}=1, \vartheta_{\max }=3$ and $\nu=\vartheta_{\max }-r_{\min }$. In addition, the iISS condition in Theorem 6 implicitly needs $\vartheta_{\max }<r_{\min }$. Another interpretation of the ISS condition of Theorem 6 is that the system (1) has local approximation at infinity $f(x, 0)$.

\section{ROBUSTNESS OF LOCALLY HOMOGENEOUS SYSTEMS}

The ISS property of locally homogeneous systems has been analyzed in [15], it was shown there that if the system (1) is locally homogeneous at 0 and $+\infty$, and all approximations and the system itself are globally asymptotically stable for $d=0$, then (1) is ISS. First we are going to propose a variant of that proof for approximation at infinity and, next, we will extend it for the systems not homogeneous with respect to $d$.

Now assume that the system is locally homogeneous at infinity. Define $\tilde{f}_{\infty}(x, d)=\left[f_{\infty}^{T}(x, d) 0_{m}^{T}\right]^{T}$.

Assumption 1. Let the vector field $\tilde{f}$ be $\left((\mathbf{r}, \tilde{\mathbf{r}}),+\infty, \tilde{f}_{\infty}\right)$ homogeneous with the weights $\mathbf{r}=\left[r_{1}, \ldots, r_{n}\right]>0, \tilde{\mathbf{r}}=$ $\left[\tilde{r}_{1}, \ldots, \tilde{r}_{m}\right]>0$ and degree $\nu>-r_{\min }$, i.e. for any $\epsilon>0$ there is a $\lambda_{\epsilon}>0$ such that $\sup _{\lambda \geq \lambda_{\epsilon}} \| \lambda^{-\nu} \Lambda_{r}^{-1} f\left(\Lambda_{r} y, \Lambda_{\tilde{r}} d\right)-$ $f_{\infty}(y, d) \| \leq \epsilon$ for all $y \in S_{r}$ and $d \in S_{\tilde{r}}$, where $f_{\infty}$ is a locally Lipschitz continuous function.

Since $\left\|\tilde{f}(x, d)-\tilde{f}_{\infty}(x, d)\right\|=\left\|f(x, d)-f_{\infty}(x, d)\right\|$, define $g(x, d)=f(x, d)-f_{\infty}(x, d)$, then by Lemma 2 in this case for all $x \in \mathbb{R}^{n}$ and $d \in \mathbb{R}^{m}$ we have

$$
\begin{aligned}
\left|g_{i}(x, d)\right| & \leq \omega_{i}\left(\|x\|_{r}+\|d\|_{\tilde{r}}\right) \\
\omega_{i}(s) & =k \begin{cases}s^{w_{\min }^{i}} & \text { if } s \leq 1 \\
s^{w_{\max }^{i}} & \text { if } s>1\end{cases}
\end{aligned}
$$

for all $i=\overline{1, n}$, where $k>0, w_{\min }^{i} \geq 0$ and $w_{\max }^{i}=r_{i}+\nu-$ $\delta<r_{i}+\nu$ for some $\delta>0$.

Theorem 8. Let Assumption 1 be satisfied. Assume that the system $\dot{x}=f_{\infty}(x, d)$ is globally asymptotically stable for $d=$ 0 , then the system (1) is ISpS.

Proof: Under the introduced conditions the system $\dot{x}=$ $f_{\infty}(x, d)$ is homogeneous with the weights $(\mathbf{r}, \tilde{\mathbf{r}})$ and the degree $\nu$, and it is globally asymptotically stable for $d=0$, then by Theorem 2 there exists a continuously differentiable, positive definite and radially unbounded Lyapunov function $V: \mathbb{R}^{n} \rightarrow \mathbb{R}_{+}$such that $V\left(\Lambda_{r} x\right)=\lambda^{\mu} V(x)$ for any $\mu>r_{\max }$ (with $\nu+\mu>0$ ), and

$$
D V(y) f_{\infty}(y, 0) \leq-a,\left\|\frac{\partial V(y)}{\partial y}\right\| \leq b \quad \forall y \in S_{r},
$$

where $a>0, b>0$. We will use the coordinate transformation $x=\Lambda_{|x|} y$ with $\Lambda_{|x|}=\left.\Lambda_{r}\right|_{\lambda=\|x\|_{r}}$, and $d=\tilde{\Lambda}_{|x|} \tilde{d}$, where $\tilde{d} \in$
$\mathbb{R}^{p}$ and $\tilde{\Lambda}_{|x|}=\left.\Lambda_{\tilde{r}}\right|_{\lambda=\|x\|_{r}}$, then the inequality (5) is satisfied. Due to homogeneity and continuity of $f_{\infty}$ with respect to $d$ we have

$$
\begin{gathered}
\left\|f_{\infty}(y, d)-f_{\infty}(y, 0)\right\| \leq \sigma(\|d\|) \quad \forall y \in S_{r}, \\
\sigma(s)= \begin{cases}c s^{\varrho_{\min }} & \text { if } s \leq 1 \\
c s^{\varrho_{\max }} & \text { if } s>1\end{cases}
\end{gathered}
$$

for some $c>0$ and $\varrho_{\max } \geq \varrho_{\min }>0$.

Now let us consider the time derivative of the Lyapunov function $V$ computed for the system (1) for all $x \in \mathbb{R}^{n}$ and $d \in \mathbb{R}^{m}$

$$
\begin{aligned}
D V(x) f(x, d) & =D V(x) f_{\infty}(x, d)+D V(x)\left[f(x, d)-f_{\infty}(x, d)\right] \\
= & \|x\|_{r}^{\nu+\mu} \frac{\partial V(y)}{\partial y} f_{\infty}(y, \tilde{d}) \\
& +\|x\|_{r}^{\mu} \frac{\partial V(y)}{\partial y} \Lambda_{|x|}^{-1}\left[f(x, d)-f_{\infty}(x, d)\right] \\
= & \|x\|_{r}^{\nu+\mu} \frac{\partial V(y)}{\partial y} f_{\infty}(y, 0) \\
& +\|x\|_{r}^{\nu+\mu} \frac{\partial V(y)}{\partial y}\left[f_{\infty}(y, \tilde{d})-f_{\infty}(y, 0)\right] \\
& +\|x\|_{r}^{\mu} \frac{\partial V(y)}{\partial y} \Lambda_{|x|}^{-1}\left[f(x, d)-f_{\infty}(x, d)\right] .
\end{aligned}
$$

Define $s(x, d)=\Lambda_{|x|}^{-1}\left[f(x, d)-f_{\infty}(x, d)\right]$, we have

$$
\begin{aligned}
\|x\|_{r}^{\nu+\mu} \frac{\partial V(y)}{\partial y} f_{\infty}(y, 0) & \leq-a\|x\|_{r}^{\nu+\mu}, \\
\|s(x, d)\| & \leq \sqrt{n} \max _{1 \leq i \leq n}\left|s_{i}(x, d)\right| \\
& \leq \tau\left(\|x\|_{r}+\|d\|_{\tilde{r}}\right), \\
\|x\|_{r}^{\mu} \frac{\partial V(y)}{\partial y}\left[f_{\infty}(y, \tilde{d})-f_{\infty}(y, 0)\right] & \leq b\|x\|_{r}^{\nu+\mu} \sigma(\|\tilde{d}\|) \\
& \leq b \gamma\left(\|x\|_{r}\right) \sigma(\|d\|),
\end{aligned}
$$

where

$$
\begin{gathered}
\gamma(s)=\left\{\begin{array}{ll}
s^{\nu+\mu-\tilde{r}_{\max } \varrho_{\min }} & \text { if } s \leq 1 \\
s^{\nu+\mu-\tilde{r}_{\min } \varrho_{\max }} & \text { if } s>1
\end{array},\right. \\
\tau(s)=\sqrt{n} k\left\{\begin{array}{ll}
s^{w_{\min }-r_{\max }} & \text { if } s \leq 1 \\
s^{w_{\max }} & \text { if } s>1
\end{array},\right. \\
w_{\min }=\min _{1 \leq i \leq n} w_{\min }^{i}, w_{\max }=\max _{1 \leq i \leq n} w_{\max }^{i}-r_{i} \leq \nu-\delta
\end{gathered}
$$

and the function $\rho$ from (5) has been used to calculate $\gamma$. From these inequalities we obtain

$$
\begin{aligned}
D V(x) f(x, d) \leq & -a\|x\|_{r}^{\nu+\mu}+b \gamma\left(\|x\|_{r}\right) \sigma\left(\|d\|_{)}\right. \\
& +b\|x\|_{r}^{\mu}\left[\tau\left(2\|x\|_{r}\right)+\tau\left(2\|d\|_{\tilde{r}}\right)\right] .
\end{aligned}
$$

By the homogeneous norm definition there exists a class $\mathcal{K}_{\infty}$ function $\chi$ such that $\tau\left(2\|d\|_{\tilde{r}}\right) \leq \chi(\|d\|)$ for all $d \in \mathbb{R}^{m}$, then

$$
\begin{aligned}
D V(x) f(x, d) \leq & -a\|x\|_{r}^{\nu+\mu}+z \kappa\left(\|x\|_{r}\right) \\
& +\left[\gamma\left(\|x\|_{r}\right)+\|x\|_{r}^{\mu}\right] \tilde{\sigma}(\|d\|)
\end{aligned}
$$

where $z=2^{w_{\max }} \sqrt{n} k b, \tilde{\sigma}(s)=b \max \{\sigma(s), \chi(s)\}$ and

$$
\kappa(s)=\left\{\begin{array}{ll}
s^{\mu+w_{\min }-r_{\max }} & \text { if } s \leq 1 \\
s^{\mu+\nu-\delta} & \text { if } s>1
\end{array}\right. \text {. }
$$


The power of $\kappa$ for the case $s>1$ is less than $\nu+\mu$ and $\mu+w_{\min }-r_{\max } \geq 0$, then $\kappa\left(\|x\|_{r}\right) \leq 1+\|x\|_{r}^{\mu+\nu-\delta}$ and we get

$$
\begin{aligned}
-a\|x\|_{r}^{\nu+\mu}+z \kappa\left(\|x\|_{r}\right) & \leq-a\|x\|_{r}^{\nu+\mu}+z+z\|x\|_{r}^{\mu+\nu-\delta} \\
& \leq-0.5 a\|x\|_{r}^{\nu+\mu}+c,
\end{aligned}
$$

where $c=z+0.5 a[1-\epsilon]^{-1}\left[2 z a^{-1}\left(1-\epsilon^{-1}\right)\right]^{\epsilon}$ and $\epsilon=(\nu+$ $\mu) / \delta$ (i.e. $-0.5 a s^{\nu+\mu}+z s^{\nu+\mu-\delta} \leq 0.5 a[1-\epsilon]^{-1}\left[2 z\left(1-\epsilon^{-1}\right)\right]^{\epsilon}$

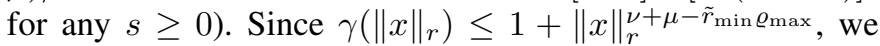
obtain

$$
\begin{aligned}
D V(x) f(x, d) \leq \quad & -0.5 a\|x\|_{r}^{\nu+\mu}+c+[1 \\
& \left.+\|x\|_{r}^{\nu+\mu-\tilde{r}_{\min } \varrho_{\max }}+\|x\|_{r}^{\mu}\right] \tilde{\sigma}(\|d\|) .
\end{aligned}
$$

Since $\tilde{r}_{\min }>0$ and $-\tilde{r}_{\min } \varrho_{\max }<0$, then from Young's inequality $\left(s v \leq \frac{s^{p}}{p}+\left(1-\frac{1}{p}\right) v^{\frac{p}{p-1}}\right.$ for any $s, v \in \mathbb{R}_{+}$and $p \geq 1$ ) we have:

$$
\begin{aligned}
s^{\nu+\mu-\tilde{r}_{\min } \varrho_{\max }} v & \leq \frac{a}{8} s^{\nu+\mu}+\pi_{1}^{-1} v^{\pi_{1}} \phi_{1}, \\
s^{\mu} v & \leq \frac{a}{8} s^{\nu+\mu}+\pi_{2}^{-1} v^{\pi_{2}} \phi_{2},
\end{aligned}
$$

where $\pi_{1}=\frac{\nu+\mu}{\tilde{r}_{\min } \varrho_{\max }}, \pi_{2}=\frac{\nu+\mu}{\nu}$ and $\phi_{i}=\left[\frac{a}{8} \frac{\pi_{i}}{\pi_{i}-1}\right]^{1-\pi_{i}}$ for $i=1,2$. Consequently,

$$
D V(x) f(x, d) \leq-0.25 a\|x\|_{r}^{\nu+\mu}+\theta(\|d\|)+c,
$$

where $\theta(s)=\tilde{\sigma}(s)+\sum_{i=1}^{2} \pi_{i}^{-1} \tilde{\sigma}(s)^{\pi_{i}} \phi_{i}$, which implies ISpS property by Theorem 1 .

For an example, consider the system:

$$
\begin{aligned}
& \dot{x}_{1}=x_{1}-x_{1}^{3}+x_{2}\left|x_{1}\right|^{0.75} d, \\
& \dot{x}_{2}=x_{2}-\left|x_{2}\right| x_{2}+\left|x_{1}\right|^{3.5}\left|x_{2}\right|^{0.125} d,
\end{aligned}
$$

which is $\left((\mathbf{r}, \tilde{\mathbf{r}}),+\infty, \tilde{f}_{\infty}\right)$-homogeneous with the weights $\mathbf{r}=$ $[1,2], \tilde{\mathbf{r}}=0.25$ and degree $\nu=2$ with $f_{\infty}(x, d)=\left[-x_{1}^{3}+\right.$ $\left.x_{2}\left|x_{1}\right|^{0.75} d-\left|x_{2}\right| x_{2}+\left|x_{1}\right|^{3.5}\left|x_{2}\right|^{0.125} d\right]^{T}$. The linearization of the system is unstable and it is hard to simulate this system in order to check its stability since it very stiff. However, since all conditions of Theorem 8 are satisfied, the system is ISpS

Corollary 4. Let all conditions of Theorem 8 be satisfied, $a>2^{w_{\max }} \sqrt{n} k b$ and $w_{\min }-r_{\max } \geq \nu$, then the system (1) is ISS.

Proof: As we can conclude from (11), under these additional (restrictive) conditions there is some $\varepsilon>0$ such that

$$
D V(x) f(x, d) \leq-\varepsilon\|x\|_{r}^{\nu+\mu}+\left[\gamma\left(\|x\|_{r}\right)+\|x\|_{r}^{\mu}\right] \tilde{\sigma}(\|d\|),
$$

since the power of the functions $\gamma$ is less than $\nu+\mu\left(\tilde{r}_{\min }>0\right)$, then there is a function $\varsigma \in \mathcal{K}$ such that for $\|d\| \leq \varsigma\left(\|x\|_{r}\right)$ we have

$$
D V(x) f(x, d) \leq-0.5 \varepsilon\|x\|_{r}^{\nu+\mu} .
$$

Corollary 5. Let a locally Lipschitz continuous vector field $f_{0}: \mathbb{R}^{n} \rightarrow \mathbb{R}^{n}$ be $\left(\mathbf{r},+\infty, f_{\infty}\right)$-homogeneous with a degree $\nu>-r_{\min }$ and asymptotically stable. If $f(x, d)=f_{0}(x)+d$, i.e. $d$ is an additive disturbance, or $f(x, d)=f_{0}(x+d)$, i.e. $d$ is a measurement noise, then the system (1) is ISpS.
Proof: Take $\tilde{\mathbf{r}}=\mathbf{r}+\nu$ for the additive disturbance case, and $\tilde{\mathbf{r}}=\mathbf{r}$ for the measurement noise. The result follows Theorem 8 .

There is a modification of Theorem 8, which skips homogeneity with respect to $d$ in Assumption 1 .

Assumption 2. Let the vector field $f$ be $\left(\mathbf{r},+\infty, f_{\infty}\right)$ homogeneous with degree $\nu>-r_{\min }$ for $d=0$, i.e. for any $\epsilon>0$ there is a $\lambda_{\epsilon}>0$ such that $\sup _{\lambda \geq \lambda_{\epsilon}}\left\|\lambda^{-\nu} \Lambda_{r}^{-1} f\left(\Lambda_{r} y, 0\right)-f_{\infty}(y, 0)\right\| \leq \epsilon$ for all $y \in S_{r}$, where $f_{\infty}$ is a locally Lipschitz continuous function. Let also for all $x \in \mathbb{R}^{n}$ and $d \in \mathbb{R}^{m}$ the inequality

$$
\begin{aligned}
& \|f(x, d)-f(x, 0)\| \leq \theta\left(\|x\|_{r}\right) \psi(\|d\|)+\phi(\|d\|), \\
& \theta(s)=\left\{\begin{array}{ll}
s^{\vartheta_{\min }} & \text { if } s \leq 1 \\
s^{\vartheta_{\max }} & \text { if } s>1
\end{array}, \vartheta_{\min } \geq 0, \vartheta_{\max } \in \mathbb{R}\right.
\end{aligned}
$$

be satisfied for some $\psi, \phi \in \mathcal{K}$ and $\nu>\vartheta_{\max }-r_{\min }$.

Define $g(x)=f(x, 0)-f_{\infty}(x, 0)$, by Lemma 2 in this case for all $x \in \mathbb{R}^{n}$ we have

$$
\begin{aligned}
\left|g_{i}(x)\right| & \leq \omega_{i}\left(\|x\|_{r}\right) \\
\omega_{i}(s) & =k\left\{\begin{array}{ll}
s^{w_{\text {min }}^{i}} & \text { if } s \leq 1 \\
s^{w_{\text {max }}^{i}} & \text { if } s>1
\end{array},\right.
\end{aligned}
$$

where $k>0, w_{\min }^{i} \geq 0$ and $w_{\max }^{i}=r_{i}+\nu-\delta<r_{i}+\nu$ for some $\delta>0$.

Theorem 9. Let Assumption 2 be satisfied. Assume that the system $\dot{x}=f_{\infty}(x, 0)$ is globally asymptotically stable, then the system (1) is ISpS.

Proof: Under the introduced conditions the system $\dot{x}=$ $f_{\infty}(x, 0)$ is $\mathbf{r}$-homogeneous with the degree $\nu$, and it is globally asymptotically stable for $d=0$, then by Theorem 2 there exists a continuously differentiable, positive definite and radially unbounded Lyapunov function $V: \mathbb{R}^{n} \rightarrow \mathbb{R}_{+}$such that $V\left(\Lambda_{r} x\right)=\lambda^{\mu} V(x)$ for any $\mu>r_{\max }$ (with $\nu+\mu>0$ ), and (10) holds for some $a>0, b>0$. Consider the time derivative of $V$ computed for (1) for all $x \in \mathbb{R}^{n}$ and $d \in \mathbb{R}^{m}$ (using the coordinate transformation $x=\Lambda_{|x|} y$ ):

$$
\begin{aligned}
D V(x) f(x, d)= & D V(x) f(x, 0) \\
& +D V(x)[f(x, d)-f(x, 0)] \\
= & D V(x) f_{\infty}(x, 0)+D V(x)[f(x, 0) \\
& \left.-f_{\infty}(x, 0)\right]+D V(x)[f(x, d)-f(x, 0)]
\end{aligned}
$$

$$
\begin{aligned}
\leq \quad & -a\|x\|_{r}^{\nu+\mu}+\|x\|_{r}^{\mu} \frac{\partial V(y)}{\partial y} \Lambda_{|x|}^{-1}[\phi(\|d\|) \\
& \left.+\theta\left(\|x\|_{r}\right) \psi(\|d\|)\right]+\|x\|_{r}^{\mu} \frac{\partial V(y)}{\partial y} s(x) \\
\leq \quad & -a\|x\|_{r}^{\nu+\mu}+\kappa\left(\|x\|_{r}\right)+\gamma\left(\|x\|_{r}\right) \psi(\|d\|) \\
& +\tau\left(\|x\|_{r}\right) \phi(\|d\|),
\end{aligned}
$$


where $s(x)=\Lambda_{|x|}^{-1} g(x), \gamma(s)=\tau \circ \theta(s)$ and

$$
\begin{aligned}
\tau(s) & =\left\{\begin{array}{ll}
s^{\mu-r_{\max }} & \text { if } s \leq 1 \\
s^{\mu-r_{\min }} & \text { if } s>1
\end{array},\right. \\
\kappa(s) & =\sqrt{n} b k s^{\mu} \max _{1 \leq i \leq n}\left|s_{i}(x)\right| \\
& =\left\{\begin{array}{ll}
s^{\mu+w_{\min }-r_{\max }} & \text { if } s \leq 1 \\
s^{\mu+w_{\max }} & \text { if } s>1
\end{array},\right.
\end{aligned}
$$

$w_{\min }=\min _{1 \leq i \leq n} w_{\min }^{i}$ and $w_{\max }=\max _{1 \leq i \leq n} w_{\max }^{i}-r_{i} \leq$ $\nu-\delta$. The powers of $\gamma, \kappa$ and $\tau$ for the case $s>1$ are less than $\nu+\mu$ (since $\mu+\vartheta_{\max }-r_{\min }<\mu+\nu$ and $\mu-r_{\min }<\mu+\nu$ for $\left.\nu>\max \left\{\vartheta_{\max }-r_{\min },-r_{\min }\right\}, \mu+w_{\max } \leq \mu+\nu-\delta<\mu+\nu\right)$, then the negative term with the power $\nu+\mu$ would dominate $\gamma, \kappa$ and $\tau$ for sufficiently high values of $\|x\|_{r}$. Next the proof repeats the technical steps of the proof of Theorem 8 .

Theorems 8 and 9 extend the conditions of theorems 5 and 6 on the case of local homogeneity at infinity. However, in the local case the difference between applicability conditions of theorems 8 and 9 is minor, the main advantage is that the local approximation at infinity may be failed to exist for both variables $x$ and $d$ (the case of Theorem 8), but it may exist for $d=0$ and Theorem 9 can be applied int this case.

\section{CONCLUSION}

Several conditions of the ISS and iISS properties have been developed based on the homogeneity theory. The advantage of these conditions is that the system robustness can be checked after its asymptotic stability in the unperturbed case provided that some algebraic homogeneity constraints are satisfied for the system equations (globally or locally). All results are obtained for generic nonlinear systems. Several examples are proposed showing efficiency of the proposed theory and its limitations.

\section{REFERENCES}

[1] J. Doyle, B. Francis, and A. Tannenbaum, Feedback Control Systems. Mac Millan Publishing Co, 1992.

[2] D. J. Hill and P. J. Moylan, "Dissipative dynamical systems: basic inputoutput and state properties," J. Franklin Inst., vol. 309, no. 5, pp. 327$357,1980$.

[3] A. van der Schaft, $L_{2}$-gain and passivity techniques in nonlinear control, vol. 218 of Lecture Notes in Control and Information Sciences. London: Springer-Verlag London Ltd., 1996.

[4] E. D. Sontag, "The ISS philosophy as a unifying framework for stabilitylike behavior," in Nonlinear control in the year 2000, Vol. 2 (Paris), vol. 259 of Lecture Notes in Control and Inform. Sci., pp. 443-467, London: Springer, 2001.

[5] M. Vidyasagar, Input-output analysis of large-scale interconnected systems, vol. 29 of Lecture Notes in Control and Information Sciences. Berlin: Springer-Verlag, 1981. Decomposition, well-posedness and stability.

[6] J. C. Willems, "Dissipative dynamical systems. I. General theory," Arch. Rational Mech. Anal., vol. 45, pp. 321-351, 1972.

[7] E. D. Sontag, "Smooth stabilization implies coprime factorization," IEEE Trans. Automat. Control, vol. 34, no. 4, pp. 435-443, 1989.

[8] S. Dashkovskiy, D. Efimov, and E. Sontag, "Input to state stability and allied system properties," Automation and Remote Control, vol. 72, no. 8, pp. 1579-1614, 2011.

[9] S. Bhat and D. Bernstein, "Geometric homogeneity with applications to finite-time stability," Mathematics of Control, Signals and Systems, vol. 17, pp. 101-127, 2005.
[10] L. Grüne, "Homogeneous state feedback stabilization of homogeneous systems," SIAM J. Control Optimization, vol. 38, no. 4, pp. 1288-1314, 2000.

[11] H. Hermes, "Homogeneous feedback controls for homogeneous systems," Systems \& Control letters, vol. 24, pp. 7-11, 1995.

[12] M. Kawski, "Nilpotent Lie algebras of vector fields," J. reine angew. Math, p. 1, 1988.

[13] L. Rosier, "Homogeneous Lyapunov function for homogeneous continuous vector field," Systems \& Control Letters, vol. 19, pp. 467-473, 1992.

[14] R. Sepulchre and D. Aeyels, "Stabilizability does not imply homogeneous stabilizability for controllable systems," SIAM J. Control Optimization, vol. 34, no. 5, pp. 1798-1813, 1996.

[15] V. Andrieu, L. Praly, and A. Astolfi, "Homogeneous Approximation, Recursive Observer Design, and Output Feedback," SIAM J. Control Optimization, vol. 47, no. 4, pp. 1814-1850, 2008.

[16] D. Efimov and W. Perruquetti, "Oscillations conditions in homogenous systems," in Proc. IFAC NOLCOS Symp., pp. 1379-1384, 2010.

[17] Y. Hong, " $\mathrm{H}_{\infty}$ control, stabilization, and input-output stability of nonlinear systems with homogeneous properties," Automatica, vol. 37, no. 7, pp. 819-829, 2001.

[18] E. Ryan, "Universal stabilization of a class of nonlinear systems with homogeneous vector fields," Systems \& Control Letters, vol. 26, pp. 177184, 1995.

[19] A. Bacciotti and L. Rosier, Lyapunov Functions and Stability in Control Theory. Springer, 2nd ed., 2005.

[20] H. Hermes, "Nilpotent approximations of control systems and distributions," SIAM J. Control Optim., vol. 24, p. 731, 1986.

[21] S. Bhat and D. Bernstein, "Finite time stability of continuous autonomous systems," SIAM J. Control Optim., vol. 38, no. 3, pp. 751-766, 2000.

[22] W. Perruquetti and S. Drakunov, "Finite time stability and stabilisation," in IEEE Conference on Decision and Control, no. 2, (Sydney, Australia), pp. 1894-1899, 2000. 